\title{
Applications of Compact Laser-Driven EUV/XUV Plasma Sources
}

\author{
Armin Bayer, Frank Barkusky, Stefan Döring, Peter Großmann, and Klaus Mann \\ Laser-Laboratorium Göttingen e.V., Hans-Adolf-Krebs-Weg 1, 37077 Göttingen, Germany \\ Correspondence should be addressed to Armin Bayer, armin.bayer@llg-ev.de
}

Received 1 February 2010; Revised 25 August 2010; Accepted 25 September 2010

Academic Editor: Ali Khounsary

Copyright () 2010 Armin Bayer et al. This is an open access article distributed under the Creative Commons Attribution License, which permits unrestricted use, distribution, and reproduction in any medium, provided the original work is properly cited.

\begin{abstract}
We present an overview on the EUV/XUV activities of the Laser-Laboratorium Göttingen based on table-top laser-produced plasma (LPP) sources. As target materials, gaseous jets of noble gases or solid Gold are employed. In order to obtain high EUV fluence, a Schwarzschild objective consisting of two spherical mirrors with Mo/Si multilayer coatings is adapted to the source. By demagnified (10x) imaging of the Au plasma, an EUV spot with a maximum energy density of $\sim 1.3 \mathrm{~J} / \mathrm{cm}^{2}$ is generated $(3 \mu \mathrm{m}$ diameter, pulse duration $8.8 \mathrm{~ns}$ ). First applications of this system reveal its potential for high-resolution modification and direct structuring of solid surfaces. Additionally, an EUV/XUV setup for structural analysis was developed. Using a gas puff target combined with a grazing incidence optics (Kirkpatrick-Baez arrangement), it offers the possibility to perform angular resolved reflectivity, diffraction, and scattering experiments. For chemical analysis of various samples, an NEXAFS setup was built, based on gaseous Krypton as a broadband emitter in the water-window range around the carbon K-edge (4.4 nm). Here, proof-of-principle for NEXAFS with lab-scaled XUV sources is given on polyimide as a reference.
\end{abstract}

\section{Introduction}

The societal demand for ever faster electronic devices requires a change in the manufacturing processes within the next few years. Microstructures like transistors on silicon chips are currently produced with deep UV lithography which uses $193 \mathrm{~nm}$ laser radiation as light source. Expanded by liquid immersion, this technique is able to generate patterns with a resolution down to about $45 \mathrm{~nm}$ [1]. Since the resolution of an optical system is limited by the utilized wavelength, there is no way for further reduction of the structure sizes with common UV lithography.

In order to fulfill the roadmap of the semiconductor industry (Moore's Law), new techniques have to be invented. One of the leading candidates is extreme ultraviolet lithography (EUVL [2]). Using reflective imaging optics on the basis of multilayer mirrors, electronic devices with structure sizes well below $45 \mathrm{~nm}$ could be manufactured at a wavelength of $13.5 \mathrm{~nm}$. For industrial lithographic systems, EUV radiation with high average power is required, which can be generated either by laser- or discharge-based plasma sources. Such EUV sources and corresponding beam steering optics are currently being developed with tremendous effort.
Besides semiconductor microlithography, there are also other applications of EUV radiation, which can strongly profit from the EUVL source and optics developments. Currently, the application of EUV radiation apart from microlithography comes more and more into focus. Main goal of our research is to utilize the unique interaction between EUV/XUV radiation and matter for probing, modifying, and structuring solid surfaces.

\section{Laser-Produced Plasma Sources at the LLG}

For EUV/XUV applications at the Laser Laboratorium Göttingen, a laser-produced EUV source was developed [35]. EUV radiation is generated by focusing an Nd:YAG laser (Innolas, fundamental wavelength $1064 \mathrm{~nm}$, pulse energy $700 \mathrm{~mJ}$, pulse duration 6-8 ns) onto a target. Currently, we are using gaseous (Oxygen, Nitrogen, Krypton, Xenon), liquid (Argon) and solid (Gold) target materials which are utilized for different applications.

For metrology purposes, we are using a gas puff target produced by a Proch-Trickl valve. The nozzle tip is located in the center of a vacuum chamber, which is evacuated below $10^{-3}$ mbar due to the low mean free path of EUV radiation at 


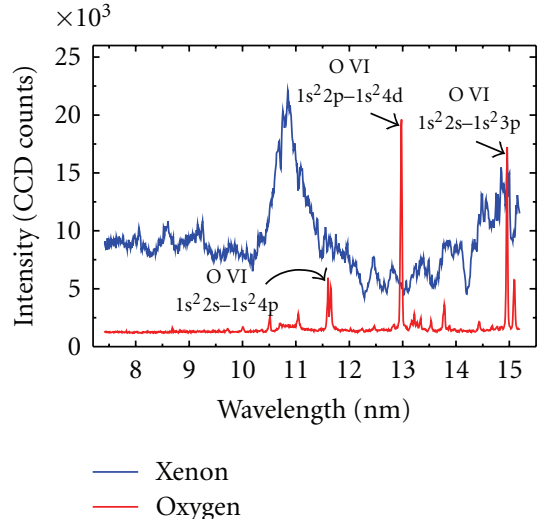

(a)

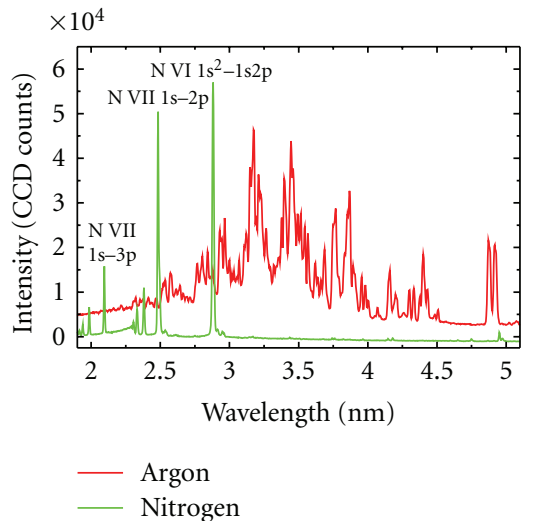

(b)

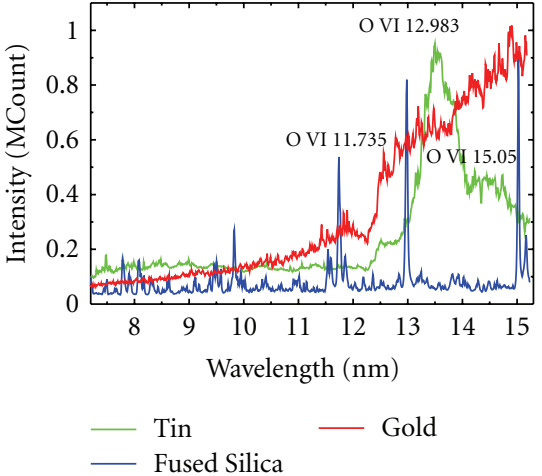

(c)

FIGURE 1: Spectra of the laser-produced plasma source developed at the LLG using different taget concepts and materials: gas puff targets in the EUV (a) and XUV (b) spectral region; solid state targets in the EUV spectral region (c). The employed target material defines the emission characteristics of the source.

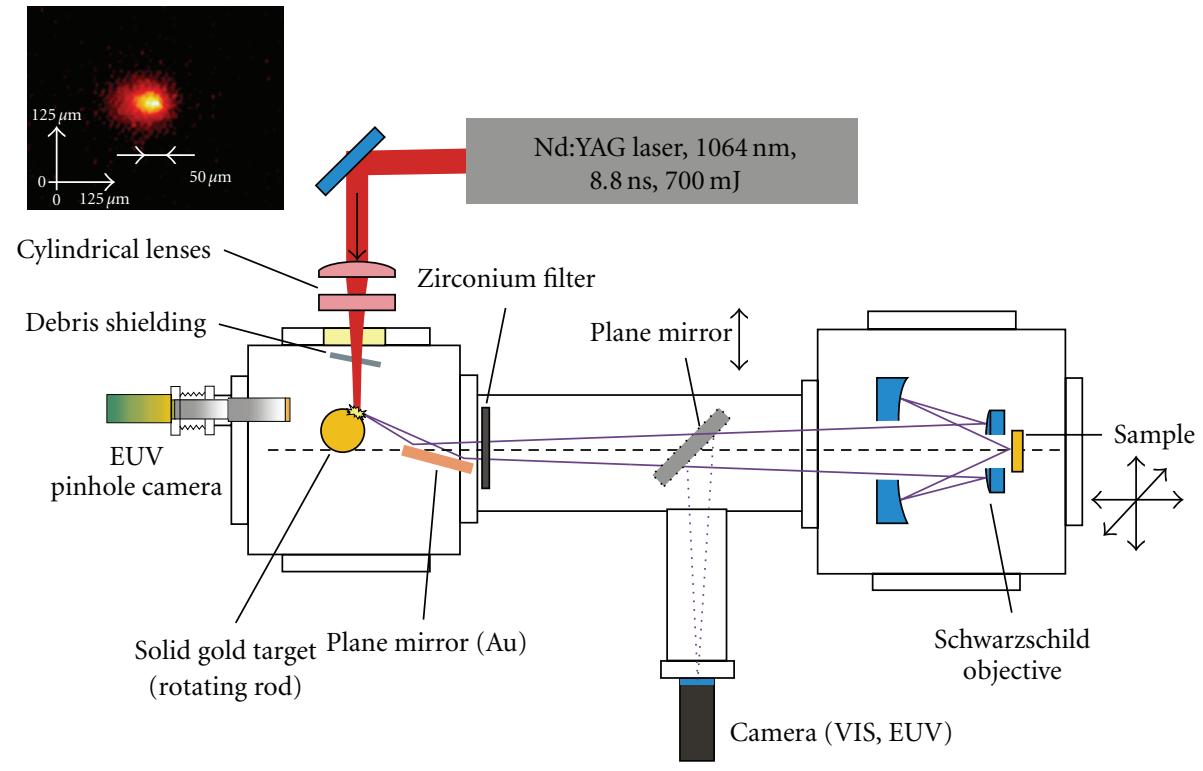

(a)

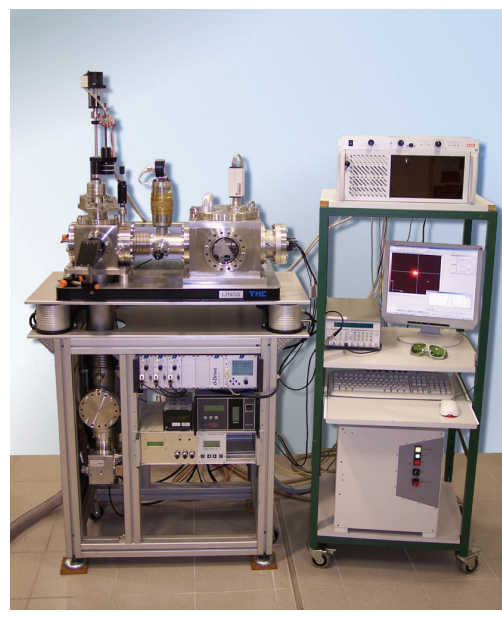

(b)

FIGURE 2: Schematic drawing of the integrated EUV source and optics system (a) and photo of the whole setup. The sample is mounted on a 3 -axes translation stage. Sample adjustment is facilitated by an integrated video camera. A pinhole camera picture is shown in the upper left.

atmospheric pressure. For applications where higher source brilliances are needed, Argon (XUV spectral range) or Gold (EUV spectral range) targets were developed (cf. Figure 1 for spectra of different target materials).

The EUV/XUV plasma can be monitored with a pinhole camera, consisting of a CCD chip with an EUV-to-VIS quantum converter and a pinhole (diameter $30 \mu \mathrm{m}$ ) coated with a zirconium filter (thickness approximately $200 \mathrm{~nm}$ ) for blocking out-of-band radiation (see also pinhole camera pictures in Figures 2 and 7).

\section{Direct Structuring and Damage Tests with EUV Radiation}

Laser ablation and photo-etching of polymers have been studied extensively especially in the visible and deep ultraviolet (DUV) spectral range [6-9]. For these wavelengths, ablation can be described in terms of thermal, photothermal, and photochemical models, or as a combination of these mechanisms [10]. A prerequisite for a preferable photochemical process without thermal load is short wavelength 


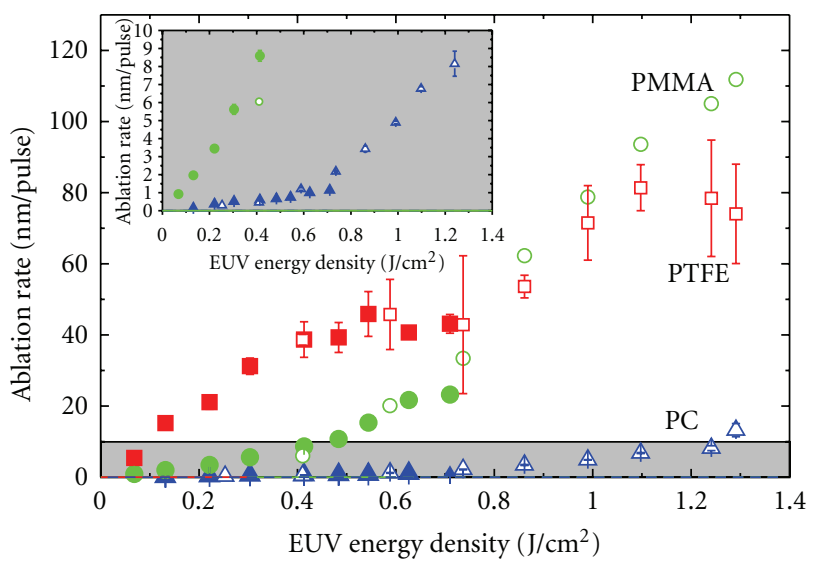

(a)

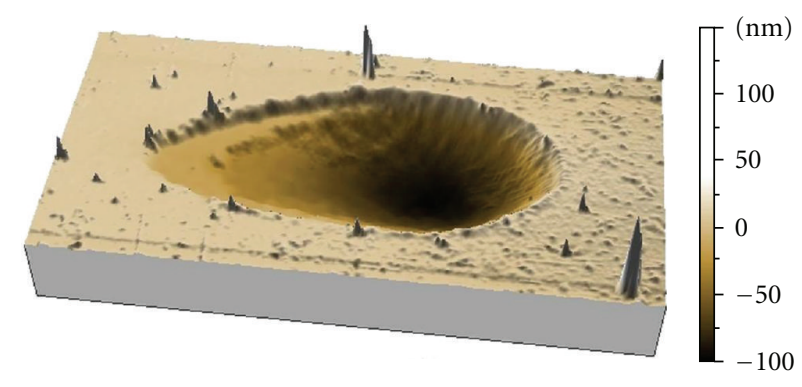

(b)

FIGURE 3: Ablation rates of various polymers, depending on the EUV energy density (a). (b) picture shows the AFM image of PMMA, irradiated with 1 EUV pulse at $1.3 \mathrm{~J} / \mathrm{cm}^{2}$.

radiation with photon energies that are high enough to directly break the polymer bonds [10]. Nevertheless, the interaction processes between polymers and radiation below a wavelength of $157 \mathrm{~nm}$ were investigated only in a few publications up to now. Examples are the structuring of PMMA using a capillary discharge Ne-like Ar laser (wavelength $46.9 \mathrm{~nm}$, pulse duration $1.2 \mathrm{~ns}$ ) [11], the freeelectron laser (FEL) in Hamburg (FLASH) emitting $32 \mathrm{~nm}$ radiation at apulse duration of $25 \mathrm{fs}$ [12], and a zinc Xray laser (wavelength $21 \mathrm{~nm}$, pulse duration $90 \mathrm{ps)} \mathrm{[13].} \mathrm{In}$ this context, it is interesting to note that at the FLASH light source, the ablation of PMMA is used also for spatial characterization of the FEL beam. Besides the EUV laser sources, also synchrotron radiation [14] and tabletop EUV/XUV sources [15-17] were used for structuring of polymers. Usually the latter are capable to generate only relatively low energy densities. Using a laser-induced Ta plasma in combination with a grazing incident $\mathrm{Au}$ covered collector mirror, an energy density of up to $0.3 \mathrm{~J} / \mathrm{cm}^{2}$ was obtained that could be applied for ablation of silica glass [18]. Unfortunately, grazing incidence mirrors are not useful for mask-projection due to their inherently low numerical aperture and corresponding low spatial resolution. In this paper, we present a table-top EUV setup which is able to generate energy densities up to $1.3 \mathrm{~J} / \mathrm{cm}^{2}$ at pulse durations of $8.8 \mathrm{~ns}$ with high spectral purity. By removing the zirconium filter $\left(\mathrm{T} \sim 17 \%\right.$ ), the fluence can be increased to $6.6 \mathrm{~J} / \mathrm{cm}^{2}$. However, due to the increased spectral bandwidth (filtering only by $\mathrm{Mo} / \mathrm{Si}$ mirrors of the objective), radiation in the visible and IR radiation might contribute to the observed damage morphologies.

3.1. Setup. The ambition of the designed EUV source and optics setup was to achieve a high energy density in the focus plane of an objective, combined with a high spatial resolution (several lines per micron) and a compact setup. For this reason, a solid Gold-based LPP source was adapted to a modified Schwarzschild objective [19]. By using additional beam-shaping techniques for the Nd:YAG laser, an energy density of $1.3 \mathrm{~J} / \mathrm{cm}^{2}$ of spectral filtered $13.5 \mathrm{~nm}$ radiation could be determined in the image plane of the Schwarzschild objective. This setup is currently used for various experiments, for example, the direct structuring of polymers or test of EUV optics and sensors.

3.2. Structuring of Polymers. For EUV lithography, photoresists based on PMMA are used. In this contribution, we have investigated the interaction between EUV radiation and polymers. Starting with ablation experiments on PMMA [20], we have expanded the study to PTFE and PC [21].

As an example, Figure 3 shows the ablation rates of the different polymers depending on the EUV energy density. Typical threshold behavior could be detected for PMMA and PC. Saturation occurs for PTFE due to the very high absorption and the resulting penetration depth of approximately $53 \mathrm{~nm}$. Independent from polymer and fluences, roughness increases due to the structuring process by less than $30 \%$, which makes this setup interesting for the generation of smooth profiles in polymers. In summary, the interaction process seems to be photochemical dominated, where the incidence EUV photons are able to break the polymer bonds until fragments can escape into vacuum.

3.3. Damage Tests on EUV Optics and Substrates. Additionally, the setup was used for determining damage thresholds for EUV optics and substrate materials [23]. As an example, results for fused silica (Suprasil 1 by Heraeus, $2 \mathrm{~mm}$ thickness) are displayed. The sample was cut into $1 \times 1 \mathrm{~cm}^{2}$ pieces and cleaned with ethanol before irradiation

In Figure 4(a), measured ablation depths are displayed as a function of the number of applied EUV pulses for selected energy densities. Obviously, a linear behaviour is observed for low intensities. However, at an energy density $>5.4 \mathrm{~J} / \mathrm{cm}^{2}$, the crater depths for 10 pulses are deeper than expected from the linear fit for lower pulse numbers. This might be caused by a radiation-induced change of the material by 


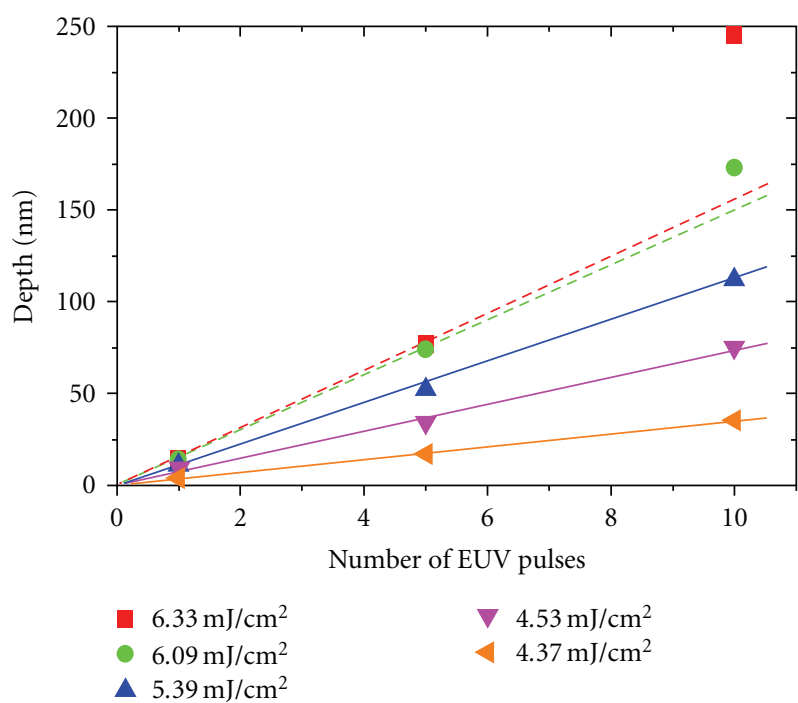

(a)

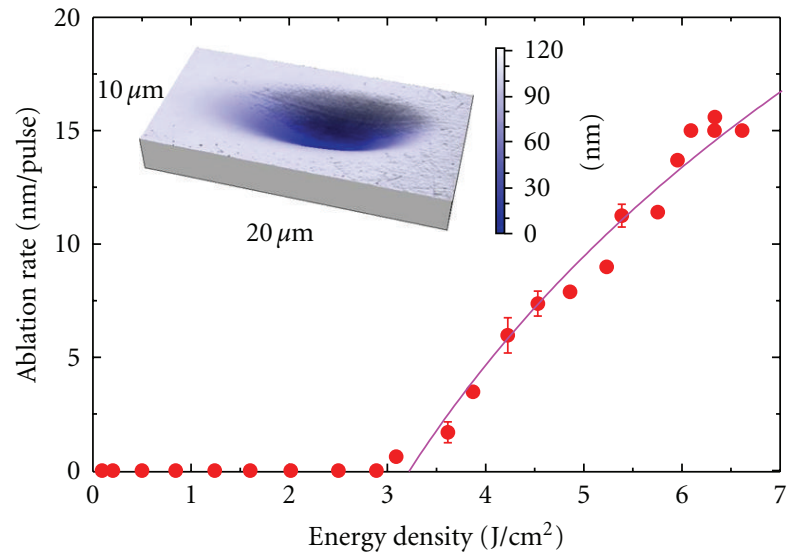

(b)

FIGURE 4: (a): Depths of EUV-generated ablation profiles in fused silica as a function of EUV pulse number for selected energy densities; (b): resulting ablation rates $d$ as a function of the applied EUV energy density $H$; the solid line represents the fit curve according to an ablation rate $d=\alpha_{\text {eff }}^{-1} \ln \left(H / H_{t}\right)\left(\alpha_{\text {eff }}=\right.$ effective absorption coefficient, $H_{t}=$ ablation threshold energy density); inset: AFM image of fused silica, irradiated with 5 pulses at highest intensity $\left(6.6 \mathrm{~J} / \mathrm{cm}^{2}\right)$.

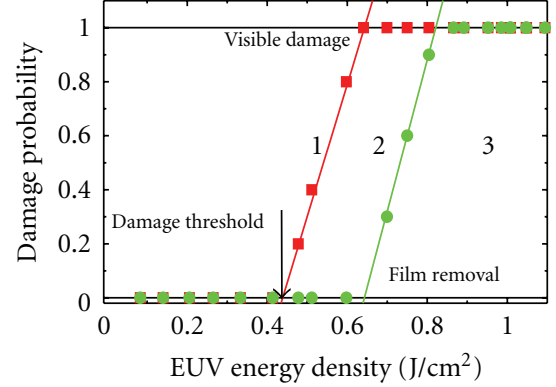

(a)

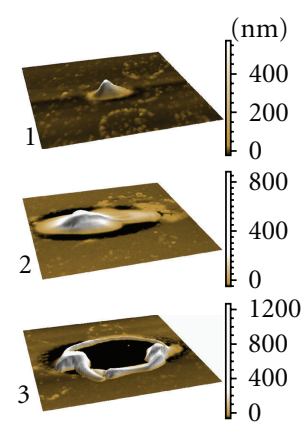

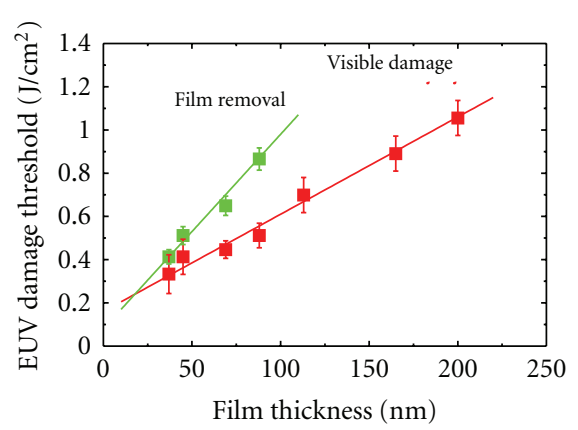

(b)

FIGURE 5: Damage threshold measurements on Gold layers. (a): Damage probability plot for a $70 \mathrm{~nm}$ thick Gold layer. Middle: corresponding AFM images. (b): film thickness dependence.

the first pulses (incubation effect). From the linear fit of the crater depths as measured with AFM, the ablation rate (depth per pulse) is determined (Figure 4(b)). The ablation rate $d$ may be fitted by a logarithmic function according to $d=\alpha_{\text {eff }}{ }^{-1} \ln \left(H / H_{t}\right)$. A corresponding best-fit yields an effective absorption coefficient $\alpha_{\text {eff }}=0.0455 \mathrm{~nm}^{-1}$ and an ablation threshold fluence $H_{t}=3.22 \mathrm{~J} / \mathrm{cm}^{2}$.

It is important to note that the absolutely smooth crater profile (see AFM micrograph) is comparable to laser ablation reported for $157 \mathrm{~nm}$ radiation with nanosecond pulses [24]. As for $157 \mathrm{~nm}$, this might be an indication for a photoninduced direct bond-breaking process, in contrast to a more thermally induced mechanism at wavelengths $>190 \mathrm{~nm}$.

For high reflectance, such fused silica substrates with for example, toroidal or ellipsoidal shapes are coated with a single (for grazing incidence reflectance) or multilayer structure (for reflectivity near to the surface nor- mal). A commonly used material for single-layer optics is Gold.

In Figure 5, the damage probability was measured for Gold films of varying thicknesses. In the left, the damage probability is plotted for a $70 \mathrm{~nm}$ thick Gold layer performing a 1-on-1 damage experiment. For this, the sample was irradiated at 10 positions with $1 \mathrm{EUV}$ pulse each at constant fluence. The number of damaged sites divided by 10 is the damage probability for this fluence. For this thin Gold layer, surface melting (1) starts at $420 \mathrm{~mJ} / \mathrm{cm}^{2}$ and complete film removal (2) at $650 \mathrm{~mJ} / \mathrm{cm}^{2}$. Repeating this experiment for different film thicknesses, (Figure 5(b)) yields a linear dependence between layer thickness and threshold energy density. This could be explained by a thermally dominated process.

Further damage experiments were performed on $\mathrm{Mo} / \mathrm{Si}$ multilayer mirrors, silicon, and calcium fluoride. For each 


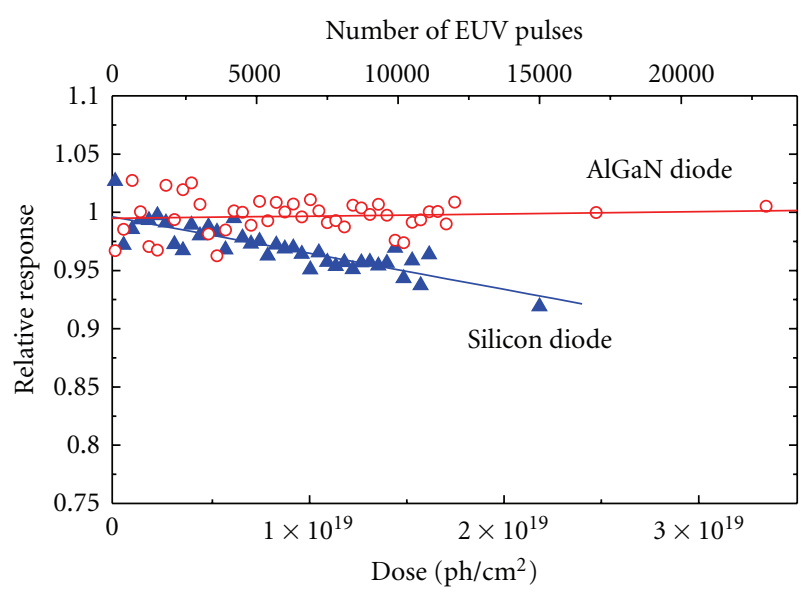

FIGURE 6: Radiation damage resistance of AlGaN diodes compared to standard silicon photodiodes.

material, damage thresholds were determined. A more detailed description of the experimental results can be found in [23].

3.4. Radiation Damage Resistance of EUV Diodes. Beside optics also damage resistance of sensors to radiation in the EUV/XUV spectral range is important, for example, in measuring absolute fluencies for EUV lithography. In this contribution, we have tested standard silicon photodiodes as well as AlGaN diodes. The latter was developed by the IMEC in Belgium and offered an intrinsically blindness to wavelengths above the UV spectral range [25]. For this kind of diode, responsivity and radiation damage resistance was measured at $13.5 \mathrm{~nm}$ wavelength. For this reason, the diodes were placed in the focus of the Schwarzschild objective. For measuring the damage resistance, the diodes were irradiated by high EUV pulse numbers. Plotting the measured responsivity against the EUV dose (EUV fluence times pulse number), the relative drop in responsivity can be displayed. The result is shown in Figure 6.

Here, a decrease in responsivity of $7 \%$ was measured for the silicon diode after $2 \cdot 10^{19}$ photons $/ \mathrm{cm}^{2}$. In contrast, for the $\mathrm{AlGaN}$ diode no change in responsivity was registered even after $3.3 \times 10^{19}$ photons $/ \mathrm{cm}^{2}$. This makes it ideal for applications where high radiation stability is needed during extended exposure to short wavelength radiation.

\section{EUV/XUV Surface Analysis Using a Reflectometry Setup}

Since extreme-ultraviolet lithography (EUVL [2]) has emerged as a leading candidate for next generation lithography in the semiconductor industry, tremendous efforts have been made to develop and optimize EUV sources and optical elements needed for the task. For beam shaping and imaging, multilayer optics are needed which have to be thoroughly characterized with respect to figure error, surface roughness, and especially reflectivity. For the latter, one of the employed techniques is angular resolved EUV reflectometry. Lacking other EUV light sources, at first this task was done mainly at synchrotron facilities such as ALS at NBNL (Berkeley) and BESSY (Berlin) and it is done there until today. But with the availability of table-top EUV/XUV light sources (laser produced and gas discharge plasma sources), optics analysis is not limited to the synchrotron facilities any more.

Still the main focus for EUV reflectometry/scatterometry setups is the characterization of EUVL components like optics and masks [26-29]. Aside from that, only little work seems to be going on with the application of EUV reflectometry for surface analysis [30].

At the LLG, the developed plasma source is employed for EUV/XUV surface analysis in a reflectometry setup. With this setup, multiple types of measurements are possible: reflectometry (in $\theta-2 \theta$-geometry), diffractometry (of nanoscale structures), and scatterometry. The wide range of measurement modes allows for analysis of a variety of sample parameters for example, refractive index, roughness, film thickness, density, and chemical composition.

4.1. Setup. The setup for EUV/XUV surface analysis is shown in Figure 7. The light emitted by the source passes through a $100 \mu \mathrm{m}$ pinhole placed approximately $2 \mathrm{~mm}$ behind the plasma and a Zirconium filter which blocks higher wavelength components of the spectra. In the optics chamber, a Kirkpatrick-Baez arrangement (cf. Figure 8) images the pinhole onto the sample placed in the middle of the experimental chamber via an $\mathrm{Mo} / \mathrm{Si}$ multilayer mirror. This mirror works as a spectral filter in the EUV spectral region and is adapted to the $1 s^{2} 2 \mathrm{p}-1 \mathrm{~s}^{2} 4 \mathrm{~d}$ emission line of Oxygen at $12.98 \mathrm{~nm}$ (cf. Figure 1(a)). The reflectometer placed in the experimental chamber consists of two independent rotation stages for sample holder and detector diode, respectively. The beam diameter on the sample (perpendicular to the incident radiation) is approximately $300 \mu \mathrm{m}$ in horizontal and $500 \mu \mathrm{m}$ in vertical direction.

For monitoring of intensity fluctuations of the plasma source, a second EUV photodiode is used as a reference monitor. It is illuminated by a second filtering multilayer mirror with the same specifications as the main mirror.

4.2. Surface Analysis Measurements. Figure 9(a) shows a basic reflectivity measurement on a $100 \mathrm{~nm}$ carbon film on silicon. As the penetration depth of EUV radiation is in the range of some $10 \mathrm{~nm}$, the curve shows quasibulk behavior. From such bulk measurements, the complex index of refraction can be calculated by line fitting the data with a mathematical model based on the Fresnel formulae.

To obtain structural information on the sample for example, film thickness and/or surface roughness, the fitting model must be expanded. For layered materials, either the Parratt Algorithm [31] or the Transfer Matrix Algorithm [32] is used and for fitting of surface roughness and/or interdiffusion thicknesses between layers commonly the Nevot-Croce Factor is used [33]. With this it is possible to obtain the film thickness of a sample fitting of the reflectometry data. Figure 9 (b) shows the measurement of a $30 \mathrm{~nm}$ carbon layer on silicon. From the oscillations that are 


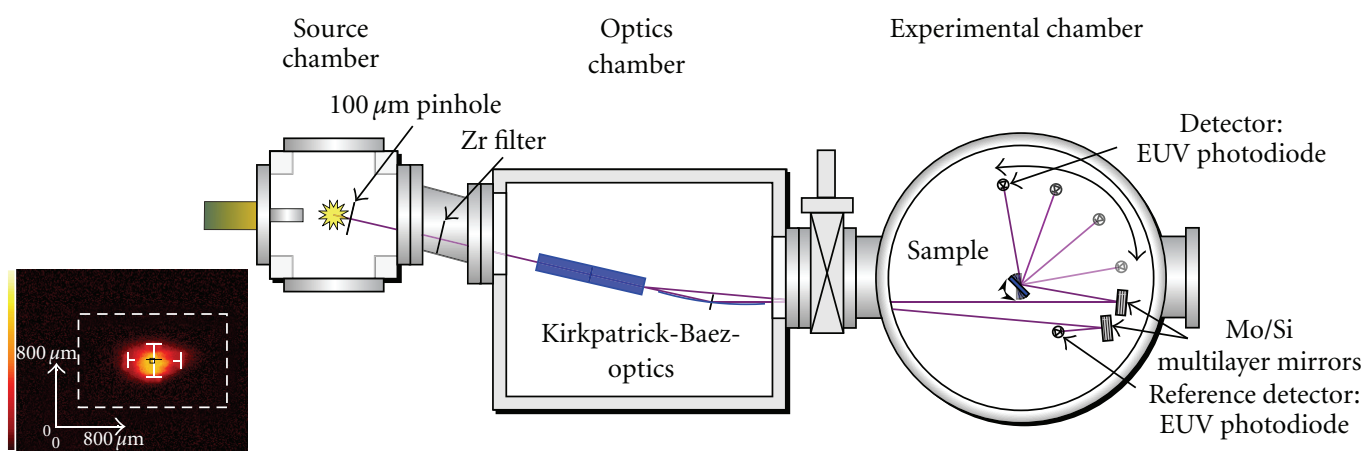

FIGURE 7: Schematic drawing of EUV/XUV surface analysis setup. It consists of an LPP source (Oxygen-target), an adaptable KirkpatrickBaez optics, and a reflectometer (cf. text). On the left, a pinhole camera image of the plasma is shown.
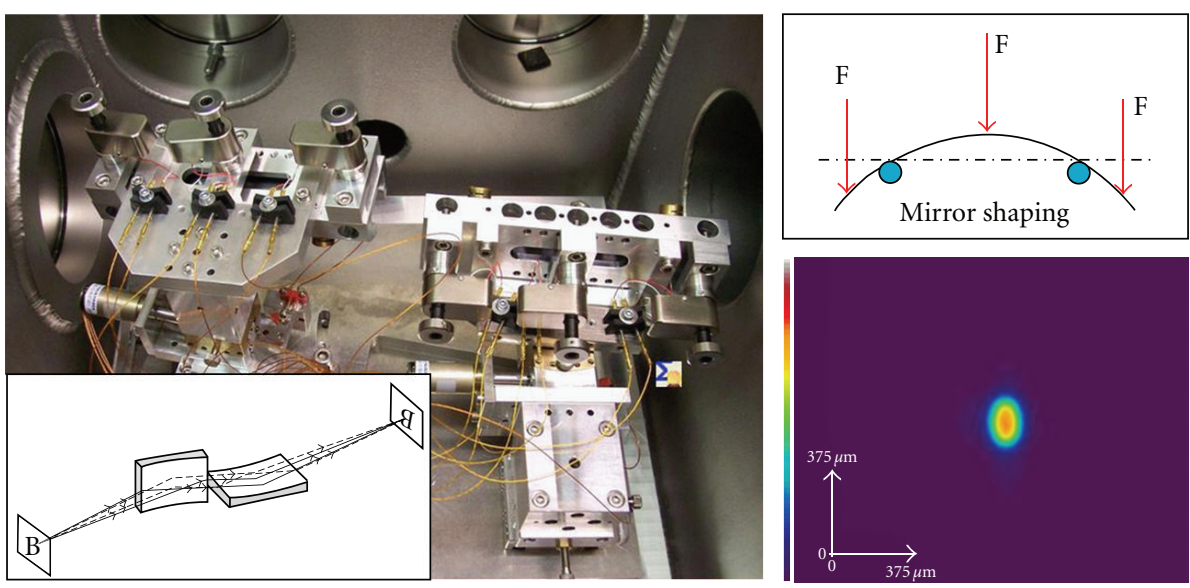

FIGURE 8: Adaptable Kirkpatrick-Baez optics used for beam shaping, based on two cylindrical bent mirrors. Left: photograph and working principle (inset). Right: principle of mirror shaping (top), using bendable silicon wafers with a high reflectivity carbon coating for beam shaping (collimating/focusing). The resulting intensity distribution (bottom) of the focused beam shows diameters of $300 \mu \mathrm{m} \times 500 \mu \mathrm{m}$ (FWHM).

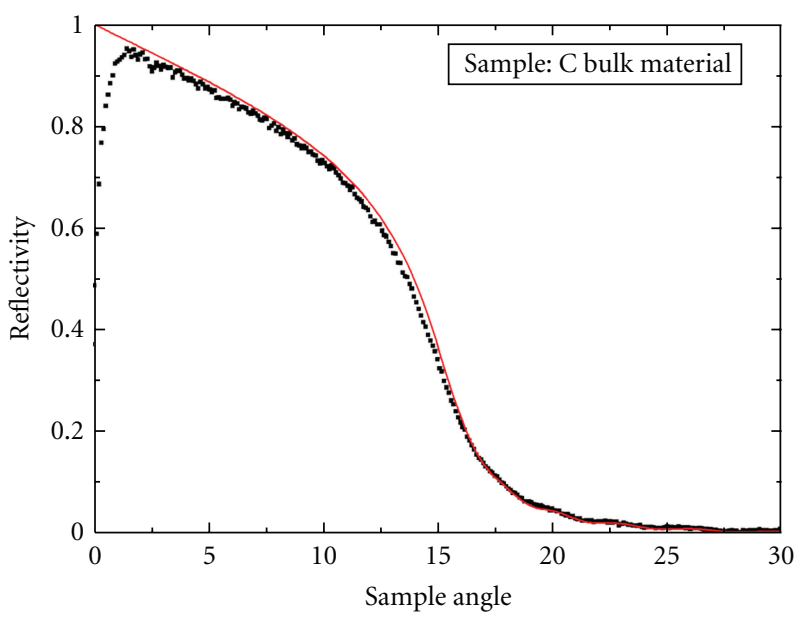

- Measurement

— CXRO@13 nm

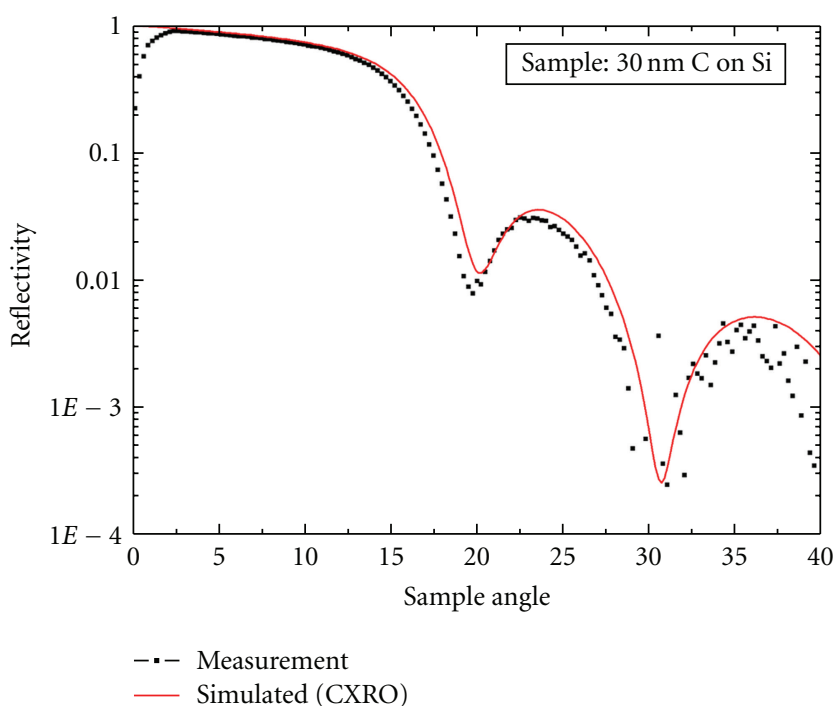

(b)

FIGURE 9: (a): Reflectivity of $100 \mathrm{~nm}$ carbon layer on silicon (black dots) compared to calculated values (red line) from the CXRO database [22]. (b): Reflectogram of a $30 \mathrm{~nm}$ carbon layer on silicon. From such measurements, the film thickness of the sample can be obtained. 


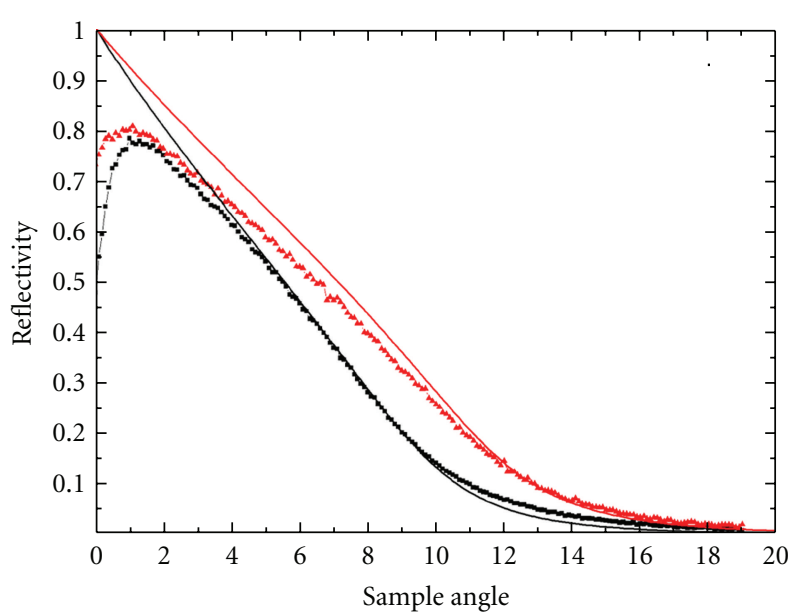

-:- Measurement $150 \mathrm{~nm} \mathrm{SiO}$ CXRO $150 \mathrm{~nm} \mathrm{SiO@13nm}$

-₫- Measurement $\mathrm{SiO}_{2}$ bulk — $\mathrm{CXRO} \mathrm{SiO}_{2}$ bulk@13 nm

(a)

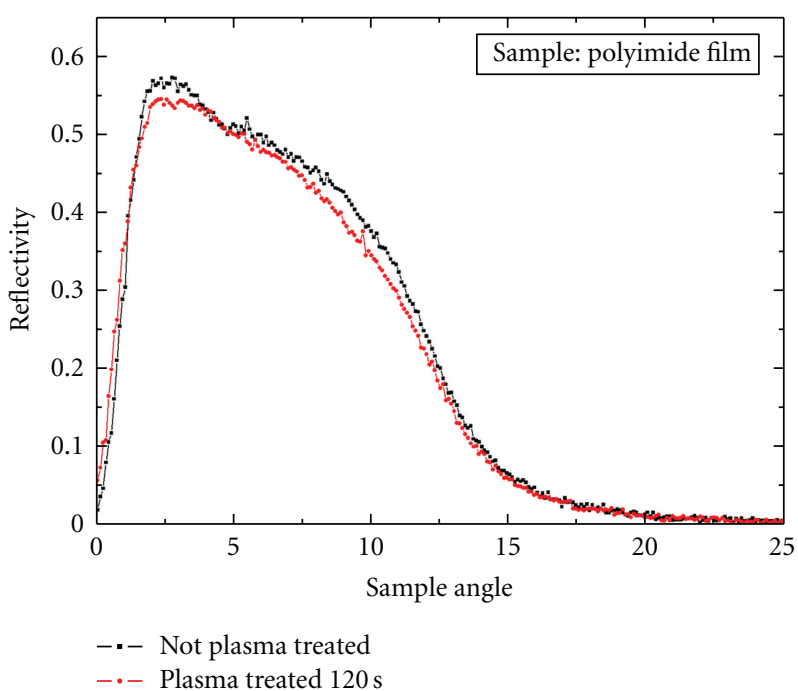

(b)

Figure 10: Material studies. (a): Reflectograms of different oxidation states of $\mathrm{Si}$ in $\mathrm{SiO}$ (black) and $\mathrm{SiO}_{2}$ (red). (b): Comparison between plasma-treated (red) and untreated (black) Polyimide foils. In spite of the small penetration depth of the plasma treatment a difference in refractive index is measurable.

due to multiple reflections in the film, the layer thickness can be obtained.

Furthermore, as the complex refractive index varies with the relative mass densities of a compound material, it is possible to distinguish different oxidation states for example, in $\mathrm{SiO}_{x}$. Figure 10(a) shows reflectograms of $\mathrm{SiO}$ and $\mathrm{SiO}_{2}$ sample measurements as well as simulations.

Even the small influence of a surface plasma-treatment on a Polyimide foil could be detected. Figure 10(b) shows the reflectograms of the plasma treated (red) and the untreated (black) samples.

\section{Near-Edge X-Ray Absorption Fine Structure (NEXAFS) Measurements}

NEXAFS, formerly used mainly for surface- and interfaceanalysis [34], is being utilized more and more in other fields, such as X-ray microscopy [35], studies on the socalled "silent" sulphur [36], or studies on transient electronic structures of for example, rare earths $[37,38]$, to only name a few. As the interest in NEXAFS grew, so the importance to develop a table-top soft X-ray source became larger. Especially for the spectral range of the "water-window" $(\lambda=2.2 \cdots 4.4 \mathrm{~nm})$ laser-driven plasma-sources have been developed in the last couple of years $[39,40]$.

In order to study the chemical structures of carbon-based specimen at the LLG, a compact setup for near edge X-ray absorption spectroscopy at the carbon K-edge based on a laser driven plasma source was built. Employing a Krypton gas puff-target, the required broad-band emission in the spectral range of the "water window" is generated. The tabletop setup consists basically of the laser plasma source and a flat-field spectrometer (Figure 11) and can be used for

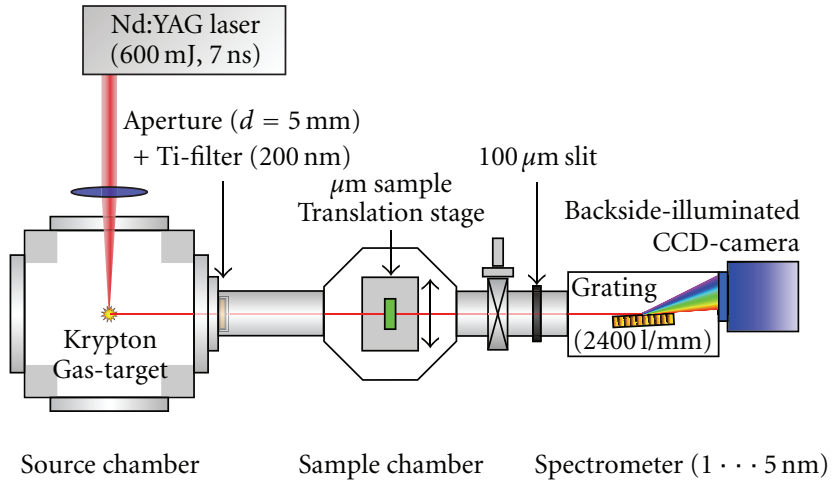

FIGURE 11: NEXAFS setup, consisting of an LPP-XUV source and an XUV flat field spectrometer.

NEXAFS experiments in transmission as well as in reflection under grazing incidence conditions (ReflEXAFS).

The latter method offers the advantage that thin film preparation is not necessary and the surface sensitivity is strongly enhanced.

In Figure 12, a measured NEXAFS spectrum of Polyimide is displayed, demonstrating the possibilities of this principle. Other applications are the determination of chemical changes in UV/EUV irradiated polymers [41] or the investigation of the transient electronic structure of PCMO.

\section{Conclusions}

The LLG table-top light source for EUV/XUV radiation is a very versatile tool for optical metrology as well as scientific applications such as surface analysis or NEXAFS. With the 


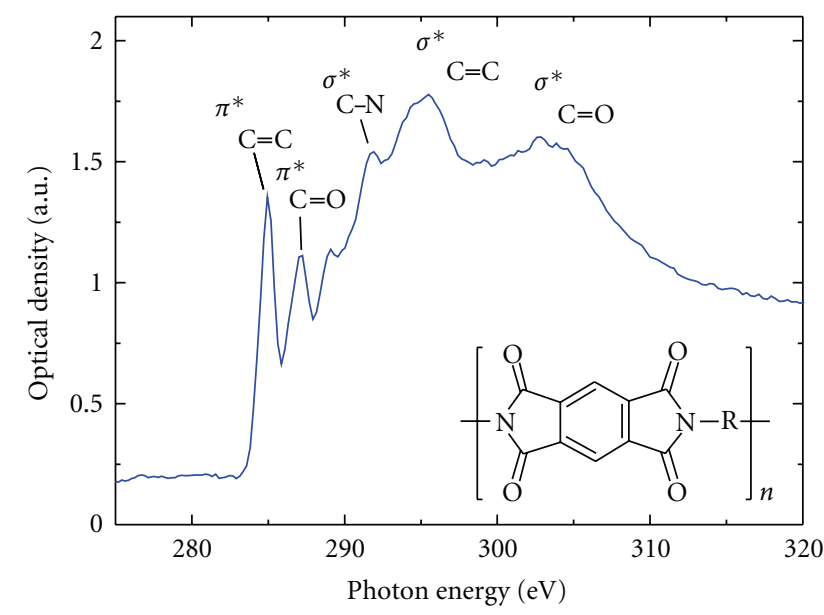

Figure 12: NEXAFS spectrum at the carbon K-edge of a Polyimide film $(d=200 \mathrm{~nm})$ measured in transmission (average over 60 pulses). The data shows a deviation of less than $0.4 \mathrm{eV}$ from corresponding synchrotron data.

help of various optical elements (Schwarzschild objective with multilayer coating for focusing, flexible KirkpatrickBaez arrangement for light guiding), the intensity distribution can be controlled, so that different experiments may be set up. The energy density within the focus of the Schwarzschild objective is sufficient to directly structure various materials such as polymers (PMMA, PC, PTFE), glasses (suprasil), crystals $\left(\mathrm{Si}, \mathrm{CaF}_{2}\right)$, or metals $(\mathrm{Au})$. From angular resolved reflectometry much information about material composition, density and surface roughness may be gained, whereas investigations on the near-edge X-ray absorption fine structure (NEXAFS) yield information about chemical bonding type and molecular orbitals.

\section{Acknowledgments}

The authors would like to thank Dr. A. Holländer (Fraunhofer IAP) for fabrication of plasma-treated polymer samples. Financial support by DFG (in the frame of SFB 755) and Federal Ministry for Economics \& Technology (within InnoNet project XUV-Nano) is gratefully acknowledged.

\section{References}

[1] A. K. Raub, A. Frauenglass, S. R. J. Brueck et al., "Imaging capabilities of resist in deep ultraviolet liquid immersion interferometric lithography," Journal of Vacuum Science and Technology B, vol. 22, no. 6, pp. 3459-3464, 2004.

[2] B. Wu and A. Kumar, "Extreme ultraviolet lithography: a review," Journal of Vacuum Science and Technology B, vol. 25, no. 6, pp. 1743-1761, 2007.

[3] S. Kranzusch and K. Mann, "Spectral characterization of EUV radiation emitted from a laser-irradiated gas puff target," Optics Communications, vol. 200, no. 1-6, pp. 223-230, 2001.

[4] C. Peth, S. Kranzusch, K. Mann, and W. Viöl, "Characterization of gas targets for laser produced extreme ultraviolet plasmas with a Hartmann-Shack sensor," Review of Scientific Instruments, vol. 75, no. 10, pp. 3288-3293, 2004.
[5] S. Kranzusch, C. Peth, and K. Mann, "Spatial characterization of extreme ultraviolet plasmas generated by laser excitation of xenon gas targets," Review of Scientific Instruments, vol. 74, no. 2, pp. 969-974, 2003.

[6] R. Srinivasan and S. Lazare, "Modification of polymer surfaces by far-ultraviolet radiation of low and high (laser) intensities," Polymer, vol. 26, no. 9, pp. 1297-1300, 1985.

[7] S. Küper, S. Modaressi, and M. Stuke, "Photofragmentation pathways of a PMMA model compound under UV excimer laser ablation conditions," Journal of Physical Chemistry, vol. 94, no. 19, pp. 7514-7518, 1990.

[8] J. Ihlemann and K. Rubahn, "Excimer laser micro machining: fabrication and applications of dielectric masks," Applied Surface Science, vol. 154, pp. 587-592, 2000.

[9] T. Lippert and J. T. Dickinson, "Chemical and spectroscopic aspects of polymer ablation: special features and novel directions," Chemical Reviews, vol. 103, no. 2, pp. 453-485, 2003.

[10] T. Lippert, "Interaction of photons with polymers: from surface modification to ablation," Plasma Processes and Polymers, vol. 2, no. 7, pp. 525-546, 2005.

[11] L. Juha, M. Bittner, D. Chvostova et al., "Ablation of organic polymers by 46.9-nm-laser radiation," Applied Physics Letters, vol. 86, no. 3, Article ID 034109, 3 pages, 2005.

[12] J. Chalupský, L. Juha, J. Kuba et al., "Characteristics of focused soft X-ray free-electron laser beam determined by ablation of organic molecular solids," Optics Express, vol. 15, no. 10, pp. 6036-6043, 2007.

[13] T. Mocek, B. Rus, M. Stupka et al., "Focusing a multimillijoule soft X-ray laser at $21 \mathrm{~nm}$," Applied Physics Letters, vol. 89, no. 5, Article ID 051501, 2006.

[14] Y. Zhang, T. Katoh, M. Washio, H. Yamada, and S. Hamada, "High aspect ratio micromachining Teflon by direct exposure to synchrotron radiation," Applied Physics Letters, vol. 67, p. 872, 1995.

[15] A. Bartnik, H. Fiedorowicz, R. Jarocki et al., "Strong temperature effect on X-ray photo-etching of polytetrafluoroethylene using a $10 \mathrm{~Hz}$ laser-plasma radiation source based on a gas puff target," Applied Physics B, vol. 82, no. 4, pp. 529-532, 2006.

[16] F. Barkusky, C. Peth, A. Bayer, and K. Mann, "Direct photoetching of poly(methyl methacrylate) using focused extreme ultraviolet radiation from a table-top laser-induced plasma source," Journal of Applied Physics, vol. 101, no. 12, Article ID 124908, 2007.

[17] H. Fiedorowicz, A. Bartnik, K. Jakubczak et al., "Application of laser plasma soft X-ray and EUV sources in micro- and nanotechnology," in Laser Technology VIII: Applications of Lasers, Proceedings of SPIE, September 2006.

[18] T. Makimura, H. Miyamoto, Y. Kenmotsu, K. Murakami, and H. Niino, "Direct micromachining of quartz glass plates using pulsed laser plasma soft X-rays," Applied Physics Letters, vol. 86, no. 10, Article ID 103111, 2 pages, 2005.

[19] F. Barkusky, C. Peth, K. Mann, T. Feigl, and N. Kaiser, "Formation and direct writing of color centers in LiF using a laser-induced extreme ultraviolet plasma in combination with a Schwarzschild objective," Review of Scientific Instruments, vol. 76, no. 10, Article ID 105102, 5 pages, 2005.

[20] F. Barkusky, C. Peth, A. Bayer, and K. Mann, "Direct photoetching of poly(methyl methacrylate) using focused extreme ultraviolet radiation from a table-top laser-induced plasma source," Journal of Applied Physics, vol. 101, no. 12, Article ID 124908, 2007.

[21] F. Barkusky, A. Bayer, C. Peth, and K. Mann, "Direct photoetching of polymers using radiation of high energy density 
from a table-top extreme ultraviolet plasma source," Journal of Applied Physics, vol. 105, no. 1, Article ID 014906, 2009.

[22] Center for X-Ray Optics, Berkeley Lab, http://wwwcxro.lbl.gov/.

[23] F. Barkusky, A. Bayer, S. Döring, P. Grossmann, and K. Mann, "Damage threshold measurements on EUV optics using focused radiation from a table-top laser produced plasma source," Optics Express, vol. 18, no. 5, pp. 4346-4355, 2010.

[24] J. Ihlemann, M. Schulz-Ruhtenberg, and T. Fricke-Begemann, "Micro patterning of fused silica by ArF- and F2-laser ablation," Journal of Physics: Conference Series, vol. 59, no. 1, pp. 206-209, 2007.

[25] F. Barkusky, C. Peth, A. Bayer, K. Mann, J. John, and P. E. Malinowski, "Radiation damage resistance of AlGaN detectors for applications in the extreme-ultraviolet spectral range," Review of Scientific Instruments, vol. 80, no. 9, Article ID 093102, 2009.

[26] J. Perlich, F.-M. Kamm, J. Rau, F. Scholze, and G. Ulm, "Characterization of extreme ultraviolet masks by extreme ultraviolet scatterometry," Journal of Vacuum Science and Technology B, vol. 22, no. 6, pp. 3059-3062, 2004.

[27] R. N. Watts, C. Tarrio, T. B. Lucatorto et al., "XUV optics characterization at the National Institute of Standards and Technology," Nuclear Instruments and Methods in Physics Research Section B, vol. 79, no. 1-4, pp. 244-246, 1993.

[28] L. Van Loyen, T. Böttger, S. Schädlich et al., "Laboratory LPP EUV reflectometer working with non-polarized radiation," Applied Surface Science, vol. 252, no. 1, pp. 57-60, 2005.

[29] S. Schröder, T. Herffurth, M. Trost, and A. Duparré, "Angleresolved scattering and reflectance of extreme-ultraviolet multilayer coatings: measurement and analysis," Applied Optics, vol. 49, no. 9, pp. 1503-1512, 2010.

[30] M. Banyay and L. Juschkin, "Table-top reflectometer in the extreme ultraviolet for surface sensitive analysis," Applied Physics Letters, vol. 94, no. 6, Article ID 063507, 2009.

[31] L. G. Parratt, "Surface studies of solids by total reflection of X-rays,” Physical Review, vol. 95, no. 2, pp. 359-369, 1954.

[32] C. C. Katsidis and D. I. Siapkas, "General transfer-matrix method for optical multilayer systems with coherent, partially coherent, and incoherent interference," Applied Optics, vol. 41, no. 19, pp. 3978-3987, 2002.

[33] P. Croce and L. Nevot, "Étude des couches minces et des surfaces par réflexion rasante, spéculaire ou diffuse, de rayons X," Revue de Physique Appliquée, vol. 11, no. 1, pp. 113-125, 1976.

[34] J. Stöhr, NEXAFS Spectroscopy, vol. 25 of Springer Series in Surface Science, Springer, New York, NY, USA, 2003.

[35] H. Ade and H. Stoll, "Near-edge X-ray absorption finestructure microscopy of organic and magnetic materials," Nature Materials, vol. 8, no. 4, pp. 281-290, 2009.

[36] F. Jalilehvand, "Sulfur: not a "silent" element any more," Chemical Society Reviews, vol. 35, no. 12, pp. 1256-1268, 2006.

[37] M. Rini, Y. Zhu, S. Wall et al., "Transient electronic structure of the photoinduced phase of $\operatorname{Pr}_{0.7} \mathrm{Ca}_{0.3} \mathrm{MnO}_{3}$ probed with soft X-ray pulses," Physical Review B, vol. 80, no. 15, Article ID 155113, 2009.

[38] M. V. Zimmermann, C. S. Nelson, J. P. Hill et al., "X-ray resonant scattering studies of orbital and charge ordering in $\mathrm{Pr}_{1-x} \mathrm{Ca}_{x} \mathrm{MnO}_{3}$," Physical Review B, vol. 64, no. 19, Article ID 195133, pp. 1951331-19513317, 2001.

[39] M. Beck, U. Vogt, I. Will et al., "A pulse-train laser driven XUV source for picosecond pump-probe experiments in the water window," Optics Communications, vol. 190, no. 1-6, pp. 317326, 2001.

[40] C. Peth, F. Barkusky, and K. Mann, "Near-edge X-ray absorption fine structure measurements using a laboratory-scale XUV source," Journal of Physics D, vol. 41, no. 10, Article ID 105202, 2008.

[41] B. Fuchs, F. Schlenkrich, S. Seyffarth et al., "Hardening of smooth pulsed laser deposited PMMA films by heating," Applied Physics A, vol. 98, no. 4, pp. 711-715, 2010. 